López Martín, I. y González Villanueva, P. (2018). La tutoría universitaria como espacio de relación personal.' Un estudio de caso múltiple. Revista de Investigación Educativa, 36(2), 381-399.

DOI: http://dx.doi.org/10.6018/rie.36.2.291161

\title{
La tutoría universitaria como espacio de relación personal. Un estudio de caso múltiple
}

\section{University tutoring as a space for personal relations. A multiple case study}

\author{
Inmaculada López Martín* y Purificación González Villanueva* \\ "Departamento de Enfermería. Facultad de Ciencias Biomédicas y de la Salud. \\ Universidad Europea de Madrid (España) \\ * Departamento de Enfermería y Fisioterapia. Facultad de Medicina y Ciencias de la Salud. \\ Universidad de Alcalá (España)
}

\begin{abstract}
Resumen
La acción tutorial implica asesoramiento académico, personal y profesional. El presente estudio tuvo como objetivo principal, indagar en las relaciones profesor-estudiante en la tutoría universitaria desde la visión del tutor, e identificar elementos de mejora. Se utilizó un diseño cualitativo. Como método, un estudio de caso múltiple, considerando "el caso": universidad pública y universidad privada. Como resultados, surgen seis temas. Presentamos "la tutoría como espacio de relación". Este tema muestra que la tutoría debe fundamentarse en la comunicación y la relación personal cercana, basada en el respeto y la privacidad. Dado que, no es una relación entre iguales, los profesores coinciden en la necesidad de establecer límites para no perder su estatus. Se concluye que, cuanto menos asimétrica y jerárquica es la relación tutor-estudiante, más efectivo es el aprendizaje. La motivación y formación del profesorado, entre otros aspectos, han de tenerse en cuenta para un modelo de tutoría sólido.

Palabras clave: universidad; tutoría; estudio de casos; relación; profesor; alumno.
\end{abstract}

Correspondencia: Inmaculada López Martín, inmaculada.lopez@universidadeuropea.es, Departamento de Enfermería, Universidad Europea de Madrid, Edificio A, 2ª planta, C/ Tajo s/n, Villaviciosa de Odón, 28670 Madrid. 


\begin{abstract}
The action of tutoring implies academic, personal and professional guidance. The main objective of this study was to deepen our understanding of the teacher-student relationships in university tutoring from the point of view of the tutor, and to identify elements for improvement. A qualitative design was used. The method employed was a multiple case study, considering "the case" as the public and private university. Six themes emerged from the data. We present "tutoring as a space for relationships". This theme shows that tutoring should be based on communication and a close personal relationship, with respect and privacy. Considering it is not a relationship among equals, teachers agree on the need to stablish limits, to avoid losing their status. In conclusion, the less asymmetric and hierarchic the tutor-student relationship is, learning becomes more effective. Motivation and teacher training must be considered, among other aspects, for a solid model of tutoring.

Keywords: university; tutoring; case study; relationship; professor; student.
\end{abstract}

\title{
Introducción
}

Una universidad que responda a las necesidades y características del siglo XXI precisa de una revisión y adecuación de la tutoría y de la figura del tutor (Lázaro, 2002). La orientación y la acción tutorial constituyen uno de los instrumentos más potentes para facilitar la transición a la universidad y promocionar a los estudiantes en la Educación Superior (Figuera \& Álvarez, 2014). En cada modelo educativo, el papel del tutor universitario ha estado en función del objetivo de la formación; de forma que los modelos de tutoría están en consonancia con la misión asumida por la universidad. Se consideran tres modelos, de acuerdo con los países que lideraron su desarrollo: el modelo alemán, centrado en la formación académica y un tipo de tutorización ligada al desarrollo de competencias en investigación; el modelo francés, orientado a la formación profesional y a la adquisición de competencias para el mundo laboral; y el modelo anglosajón, basado en una orientación integral y personalizada de los estudiantes (García Nieto, 2008; Lázaro, 2002; López-Gómez, 2015; Torrecilla, Rodríguez, Herrera \& Martín, 2013). Acorde con este modelo anglosajón que adopta el EEES, la tutoría se define como un proceso formativo, de orientación integral y acompañamiento, desarrollado por el profesorado, que implica acciones para proporcionar a los estudiantes la ayuda necesaria para conseguir sus objetivos académicos, personales y profesionales. En definitiva, atender las necesidades que les plantea la universidad (López-Gómez, 2015; Martínez, Pérez \& Martínez, 2014); donde el profesor debe asumir un papel de facilitador, de forma que los estudiantes sean los protagonistas de su aprendizaje (López-Gómez, 2015; Pantoja \& Campoy, 2009; Romero, Ortega \& Molina, 2016); especialmente durante el primer año de universidad, dadas las tasas de abandono en determinadas áreas de conocimiento (Pantoja \& Campoy, 2009) y los niveles de absentismo en las aulas (De Jorge, Gil, Merino \& Sanz, 2011).

En el contexto español, la tutoría ha constituido en general, una intervención puntual del profesor, cercana al modelo alemán y con el objetivo de solventar dudas académicas. En el alumnado, la tutoría académica se percibe como una ayuda com- 
plementaria a las asignaturas, sin considerarla algo central en su formación (García González \& Troyano, 2009; Rodríguez-Hoyos, Calvo \& Haya, 2015). Sin embargo, el marco que establece la reforma de la Educación Superior implica una tutoría más compleja y poliédrica, de asesoramiento continuado, que atiende el plano académico, personal y profesional; basada en la relación más cercana entre profesor y estudiante.

En las universidades españolas conviven varios tipos de tutoría, con diferentes objetivos y niveles de intervención, similares a los que describen Figuera y Álvarez (2014) y Rodríguez Espinar(2004), refiriéndose a la tutoría académica o de asignatura, tutoría de carrera o de acompañamiento y tutoría de asesoramiento personal. Existen diferencias entre las universidades públicas y privadas, de forma que en estas últimas, se admite mejor y se aborda con menor dificultad la tutoría personal y los profesores expresan menos problemas en la realización de la tutoría no estrictamente docente (Fernández \& Escribano, 2009; Giménez, 2007; Lázaro, 2002; López-Martín, González \& Velasco, 2013). Una investigación en universidades de quince países, españolas y europeas, indica que la tutoría es fundamentalmente académica (GarcíaNieto, Asensio, Carballo, García-García \& Guardia, 2005). Estudios más recientes en la universidad pública, siguen revelando la primacía de la tutoría de contenido académico (López-Gómez, 2016; Martínez, Martínez \& Pérez, 2014; Romero et al., 2016).

Cabe preguntarse si los docentes están suficientemente formados en habilidades comunicativas y de entrevista para dar respuesta a las diferentes necesidades y tipología de estudiantes en el marco de una tutoría integral. Álvarez González y Álvarez Justel (2015), destacan las debilidades de los actuales modelos de tutoría en diversas universidades, y entre ellas el escaso compromiso del profesorado y la institución para asumir el rol de tutor y la escasa formación y sensibilización para desempeñar esta función. Esta problemática se identifica también en otros países, como en el estudio de revisión bibliométrica de los programas de tutoría y mentoría en universidades brasileñas, entre 1998 y 2013, realizado por Albanaes, Marques de Sousa y Patta (2015). Destaca la poca disponibilidad de los docentes para la práctica de la mentoría y la falta de capacitación y sensibilización de los docentes para actuar como mentores/tutores. El grado de implicación de los estudiantes en su propia formación, desde el punto de vista del profesorado es bajo; y ello se evidencia en su escaso interés en las actividades universitarias, en el aula y en la utilización de la tutoría (Martínez, Pérez, et al., 2014; Paricio, 2005). Albanaes et al. (2015), también aluden a la falta de interés de los estudiantes hacia la tutoría, cuestión identificada en el contexto español. Por otro lado resulta contradictorio, que los estudiantes le concedan gran valor a la tutoría, y opinen que deba integrarse en la docencia y en los itinerarios formativos (Romero et al., 2016).

Por ello, es importante profundizar en cómo influye el perfil del tutor, su motivación, formación y forma de relacionarse en el entorno de la tutoría. Mena-Rodríguez (2015), estudia en el alumnado de cinco ramas de conocimiento, la percepción sobre el comportamiento interpersonal de los mejores docentes, concluyendo que, aspectos como el liderazgo, la comprensión y el apoyo son los que más valoran en sus profesores; y la incertidumbre, sanción o reprobación e insatisfacción, obtienen las valoraciones más bajas. En el estudio de Haya, Calvo y Rodríguez (2013), el alumnado expresa su predilección por estilos docentes en los que se establecen un tipo de 
relaciones pedagógicas basadas en la cercanía y apertura entre docente y discente. La relación profesor-alumno, está en la base del éxito o del fracaso de muchos estudiantes en su paso por cualquier modalidad formativa y/o educativa. "El tutor debe conocer a conciencia a sus alumnos, ser capaz de despertar en ellos las ganas de aprender, contagiarles entusiasmo, y ser consecuente y respetuoso" (García Fernández, 2006, p. 197).

Para desempeñar el papel de tutor, es necesaria la adquisición de competencias, entre las cuales destacan la comunicación, la capacidad para establecer relaciones personales, fundamentales para crear un buen ambiente de aprendizaje (García Fernández, 2006; Mena-Rodríguez, 2015) y habilidades concretas para el desarrollo de la tutoría (García González \& Troyano, 2009; García-Cabrero et al., 2016; Lázaro, 2008; Zabalza, 2003). Respecto a la dimensión de comunicación durante la tutoría, García-Cabrero, et al. (2016), concluyen que es importante establecer una relación horizontal, es decir, una comunicación respetuosa y empática con el tutorando, manteniendo una congruencia entre el lenguaje corporal y el verbal.

Nos preguntamos, hasta qué punto el tipo de relación entre tutores y estudiantes en la universidad, influye en el uso que los estudiantes hacen de la tutoría en general, de que depende el tipo de relación establecida, y si es diferente en universidades públicas y privadas.

A partir de estas cuestiones, nos planteamos los siguientes objetivos:

- Indagar en las relaciones profesor-estudiante en el contexto de la tutoría universitaria de cualquier tipo, desde la visión del tutor.

- Identificar como influye el tipo de relación tutor-estudiante en el uso de la tutoría.

- Identificar elementos para el desarrollo y mejora de la acción tutorial universitaria.

\section{Método}

\section{Diseño}

Este trabajo forma parte de una investigación más amplia, dirigida a conocer los significados de la tutoría en la universidad desde la percepción de los profesores (López-Martín, 2012). Se inscribe en un marco epistémico interpretativo y metodología cualitativa en el ámbito educativo.

En el área de la educación, la investigación cualitativa está orientada a la comprensión en profundidad de fenómenos educativos y sociales.

Se utilizó el estudio de casos como método de investigación. Se puede considerar un caso, una persona, un programa, un grupo de alumnos, un movimiento de profesionales, una institución; en definitiva, un sistema integrado, que debe tener unos límites (Stake, 1999). Consiste en una descripción y análisis de unidades sociales o entidades educativas únicas, desde lo heurístico y con método inductivo (Martínez, 2006). La unidad de análisis es la tutoría universitaria; es investigada a través de dos casos, el caso universidad pública (UPU) y el caso universidad privada (UPRI); por lo cual es un estudio instrumental de casos (López-Martín, 2012). Al utilizar más de un caso, también estamos ante un estudio de caso múltiple (Stake, 1999). 
El estudio se desarrolló desde agosto de 2009 hasta febrero de 2012. Se siguió el esquema de investigación adaptado de Rodríguez-Gómez, Gil-Flores y García-Jiménez (1999). Consiste en cuatro fases solapadas:

1) fase preparatoria

2) trabajo de campo

3) fase de análisis y

4) fase final informativa

\section{Contexto del estudio: casos y escenarios}

El caso UPRI estuvo formado por un único escenario, una universidad privada, seleccionada por disponer de variedad de titulaciones, desempeño de acción tutorial y fácil acceso al campo. En este escenario se realizaron múltiples observaciones en diferentes espacios semipúblicos. El caso UPU se desarrolló en tres universidades públicas como escenarios, con planes de acción tutorial en marcha. En la selección de escenarios de la UPU, se tuvieron en cuenta los siguientes criterios:

- Universidad de reconocido prestigio investigador, nombrada como "universidad tecnológica".

- Universidad con tradición, llamada "universidad antigua".

- Universidad joven y que tuviera poco recorrido en el ámbito de la Educación Superior, denominada "universidad nueva".

En ambos casos se contemplan profesores de diferentes perfiles y áreas de conocimiento (Tabla 1). Estos escenarios se encuentran en la Comunidad de Madrid, España.

Tabla 1

Distribución de escenarios en ambos casos de estudio

\begin{tabular}{|c|c|c|c|}
\hline $\begin{array}{c}\text { CASO “UNIVERSIDAD } \\
\text { PRIVADA" }\end{array}$ & \multicolumn{3}{|c|}{ CASO “UNIVERSIDAD PÚBLICA” } \\
\hline Escenario único & $\begin{array}{c}\text { Escenario 1: } \\
\text { Universidad Nueva }\end{array}$ & $\begin{array}{c}\text { Escenario 2: } \\
\text { Universidad Tecnológica }\end{array}$ & $\begin{array}{c}\text { Escenario 3: } \\
\text { Universidad } \\
\text { Antigua }\end{array}$ \\
\hline $\begin{array}{l}\text { Varios periodos de } \\
\text { observación } \\
\text { Nov. } 2010 \text { a Abril } 2011 \\
\text { (7 entrevistas) }\end{array}$ & $\begin{array}{l}\text { Dos períodos de } \\
\text { observación } \\
\text { Nov. } 2010 \\
\text { (2 entrevistas) }\end{array}$ & $\begin{array}{l}\text { Dos periodos de } \\
\text { observación } \\
\text { Febrero } 2011 \\
\text { (1 entrevista) }\end{array}$ & $\begin{array}{c}\text { Dos periodos de } \\
\text { observación } \\
\text { Enero } 2011 \\
\text { (2 entrevistas) }\end{array}$ \\
\hline
\end{tabular}

\section{Sujetos participantes}

La selección de los profesores se realizó mediante muestreo intencionado (Ruiz Olabuénaga, 2012), siguiendo el "muestreo teórico" (Glaser \& Strauss, 2009); en función 
del potencial de la muestra, para ayudar a refinar o expandir los conceptos, de forma que sus diferentes disciplinas, edades, formación, experiencia docente y experiencia como tutor, aportaran puntos de vista diversos. Por lo tanto, se incluyeron profesores de cualquier rama de conocimiento, que desempeñaban la función de tutor/mentor de estudiantes, con al menos seis meses de experiencia en el rol de tutor y cuya participación era voluntaria.

Los informantes clave para acceder a la muestra, eran responsables de Programas de Tutoría o gestores académicos. En el caso de un profesor, la muestra no partió del investigador, por lo que se asemeja al muestreo en "bola de nieve" (Goodman, 2011). Las citas para las entrevistas fueron programadas directamente con los participantes.

Se reunió una muestra de 12 profesores (7 mujeres y 5 hombres). Se obtuvo consentimiento verbal y por escrito, previo a la realización de las entrevistas. Edades comprendidas entre 25 y 64 años (media de 39,9) y experiencia como profesor entre 6 meses y 29 años (media 9,96). En el caso de la UPU participaron cinco profesores, que pertenecían a alguno de los tres escenarios. En la UPRI participaron siete profesores. Las áreas de conocimiento fueron Ciencias de la Salud (4 tutores), Ciencias Empresariales (1 tutor), Derecho (1 tutor), Deporte (2 tutores), Ciencias Físicas (2 tutores), Ciencias (1 tutor) y Área Politécnica (1 tutor). El reclutamiento de participantes finalizó cuando se produjo saturación en los datos.

Durante el trabajo de campo se realizaron diferentes acciones para la comunicación con los participantes, la explicación del proyecto y la documentación requerida del consentimiento informado.

\section{Procedimientos de recogida de datos}

Se realizó mediante entrevistas semiestructuradas, observación no participante y diario de investigación.

Las entrevistas semiestructuradas se describen como "no directivas, no estructuradas, no estandarizadas y abiertas" (Taylor \& Bogdan, 2009, p.101). Se realizaron 12 entrevistas; 5 de ellas en los despachos de los profesores de las UPUs, 7 en salas privadas o aulas vacías en la UPRI. La duración osciló entre media hora y una hora, precedida cada una de ellas por dos horas de observación del escenario. Inmediatamente después de cada entrevista, se registraron reflexiones sobre aspectos emergentes en la entrevista, que posteriormente se incorporaron al diario del investigador.

El esquema de las entrevistas siguió los cuatro bloques recomendados por Coller (2000), en el marco del estudio de casos: introducción de la persona que investiga, preguntas que contribuyan a romper el hielo, desarrollo de la entrevista guiada por preguntas sobre el tema, y finalmente, conclusión y cierre. Para cumplir con los criterios de fiabilidad y validez de los datos, hemos tenido en cuenta el concepto de credibilidad (Vallés, 2009, p.83); basándonos en la transparencia, consistenciacoherencia, y la comunicabilidad. Las preguntas iniciales relacionadas con el estudio fueron: ¿Cómo diría que es el proceso de comunicación entre profesor-alumno durante las tutorías?, ¿Qué rasgos del tutor le parecen que pueden influir o influyen en que un alumno decida solicitar una tutoría?, ¿Cómo se produce la relación/comunicación durante la tutoría?, ¿Qué influye a la hora de que un estudiante le solicite tutoría 
a su tutor?. Estas preguntas están en consonancia con los objetivos principales del estudio, y traducen las cuestiones de investigación en preguntas de entrevista o asuntos de conversación (Vallés, 2009). Siguiendo el muestreo teórico, el guion de preguntas fue evolucionando y ajustándose según la información de los participantes (Strauss \& Corbin, 2002).

Todas las entrevistas fueron realizadas por la misma investigadora, con experiencia en realización de entrevistas cualitativas (Vallés, 2009), y grabadas en formato digital.

La observación no participante fue un método de recogida de datos complementario a las entrevistas, con fines de triangulación, limitada en el tiempo, entre septiembre 2010 y febrero 2011; y documentada en el diario del investigador. Consiste en observar a participantes en su entorno, sin que el investigador participe de forma activa (Atkinson \& Hammersley, 2008). El objetivo fue observar el tipo de relación entre profesores y estudiantes, fuera del contexto de la tutoría, en espacios abiertos y/o semipúblicos, sin manipularlos ni modificarlos, permitiendo el desarrollo de los acontecimientos espontáneamente (Ruiz Olabuénaga, 2012). Se observaron actitudes y posturas, el uso del espacio para relacionarse y aspectos no verbales de la comunicación. Al observarse espacios semipúblicos, no se precisó solicitud de permisos. Las observaciones registradas en el diario de campo han formado parte del análisis con fines de rigor y credibilidad de los datos.

8 de noviembre, 2010: Me parece importante reseñar en este diario, que las interpretaciones sobre lo observado pueden ser sumamente engañosas si no se contrastan por otros medios, o con la insistencia en la observación. He ahí la importancia de la triangulación de métodos para investigar, (...). (Diario de campo)

Durante el estudio de casos, el investigador registró los acontecimientos para ofrecer una descripción exhaustiva, con el fin de realizar posteriores análisis y el informe final (Stake, 1999). Con este fin, se utilizaron cuadernos y grabadora para las notas de campo. Además, la investigadora principal redactó un diario de investigación a modo de relato personal descriptivo y reflexivo, para mantener un posicionamiento lo más neutral posible y reflejar situaciones de "extrañamiento" ante hallazgos de datos nuevos (Velasco \& Díaz de Rada, 2003, p. 216).

\section{Análisis de los datos}

Se realizó una transcripción completa y literal de las entrevistas. La lectura y análisis preliminar de las transcripciones se simultaneó con la realización de las entrevistas, las observaciones y la escritura del diario; lo cual permitió el muestreo teórico y la triangulación de los datos.

Los datos fueron analizados por dos investigadores con experiencia en estudios cualitativos. Todo el proceso de análisis fue inductivo, no se partió de categorías previas, como es habitual en los estudios de caso (Stake, 1999).

El método se basó en el análisis "línea por línea" (Strauss \& Corbin, 2002). En un primer nivel, los conceptos identificados (códigos) se mantuvieron muy "pegados" al sentido de los informantes, posteriormente se refinaron y redujeron, a través de 
un análisis reflexivo "de ida y vuelta", siguiendo el método comparativo constante (Glaser \& Strauss, 2009); combinado con los dos métodos de análisis que Stake (1999), propone en los estudios de casos: la interpretación directa de los datos y la suma categórica de todos ellos a partir de las diferentes fuentes de información para cada uno de los casos.

En un segundo nivel y en cada caso, se agruparon los códigos en categorías, y estas en metacategorías. De la relación entre las metacategorías, surgen los temas conductores en el caso UPU y UPRI. Finalmente, en un tercer nivel, se identificaron temas comunes y diferenciales en ambos, resultando los temas transversales.

El tratamiento de los datos se realizó con el programa Atlas-ti versión 5.0. El software facilitó la gestión de códigos, memorandos o conceptos sensitivos (Atkinson \& Hammersley, 2008), agrupación de categorías y generación de mapas conceptuales.

\section{Rigor}

Se realizó triangulación de datos a partir de las entrevistas, notas de campo, observación, diario, investigaciones afines y expertos en metodologías cualitativas; con el fin de tener una visión más completa del fenómeno a estudiar y contribuir a la veracidad de los datos. Tras las entrevistas se procedió a la verificación de las transcripciones por parte de los participantes. Se realizó triangulación entre investigadores en el proceso de análisis de datos, de manera que hubiera coherencia entre los resultados y las percepciones que los sujetos tenían sobre el fenómeno estudiado (Rodríguez-Gómez et al., 1999; Sandín, 2010). Solo el profesor de la universidad tecnológica realizó cambios en la transcripción de su entrevista. El diario del investigador contribuyó al proceso de reflexividad y el programa Atlas-ti permitió una mayor exhaustividad en el proceso de análisis.

\section{Aspectos éticos de la investigación}

La protección de datos de personas e instituciones se ha tenido en cuenta durante toda la investigación. Los nombres de los profesores son ficticios. Se utilizó un formato elaborado ad hoc para el consentimiento informado de los participantes. Se solicitó consentimiento escrito para la grabación de las entrevistas y permiso a las instituciones para visitar los escenarios universitarios.

\section{Resultados}

\section{Resultados globales}

Se seleccionaron 917 fragmentos de texto, a partir de los cuales se generaron 1063 códigos. La agrupación de los códigos en el caso UPU generaron 20 categorías procedentes de los tres escenarios, y estas a su vez formaron 8 metacategorías (Tabla 2). En el caso UPRI, se identificaron 7 categorías, agrupándose en 4 metacategorías, procedentes del único escenario (Tabla 3). 
Tabla 2

Resultados Caso universidad pública

\begin{tabular}{|c|c|c|c|}
\hline $\begin{array}{l}\text { CATEGORÍAS } \\
\text { UNIVERSIDAD } \\
\text { NUEVA }\end{array}$ & $\begin{array}{l}\text { CATEGORÍAS } \\
\text { UNIVERSIDAD } \\
\text { ANTIGUA }\end{array}$ & $\begin{array}{l}\text { CATEGORÍAS } \\
\text { UNIVERSIDAD } \\
\text { TECNOLÓGICA }\end{array}$ & $\begin{array}{c}\text { CASO } \\
\text { UNIVERSIDAD } \\
\text { PÚBLICA: } \\
\text { METACATEGORÍAS }\end{array}$ \\
\hline $\begin{array}{l}\text { Claves en el } \\
\text { momento actual de la } \\
\text { universidad pública. }\end{array}$ & $\begin{array}{l}\text { - La universidad } \\
\text { pública: formas } \\
\text { nuevas en moldes } \\
\text { clásicos } \\
\text { - Cuestionamiento } \\
\text { del Plan Bolonia }\end{array}$ & $\begin{array}{l}\text { El Plan Bolonia: de la } \\
\text { Teoría a la Práctica }\end{array}$ & $\begin{array}{c}\text { Aires de cambio en la } \\
\text { universidad. }\end{array}$ \\
\hline $\begin{array}{l}\text { Ser profesor en el } \\
\text { cambio del modelo de } \\
\text { universidad }\end{array}$ & $\begin{array}{l}\text { Ser profesor en la } \\
\text { universidad: entre } \\
\text { la transmisión del } \\
\text { conocimiento y la } \\
\text { investigación }\end{array}$ & $\begin{array}{l}\text { El profesor de } \\
\text { universidad ante el } \\
\text { cambio de paradigma } \\
\text { docente }\end{array}$ & $\begin{array}{l}\text { Ser y Ejercer de } \\
\text { profesor en la } \\
\text { universidad de hoy }\end{array}$ \\
\hline $\begin{array}{l}\text { El Modelo de Tutoría } \\
\text { que acompaña al Plan } \\
\text { de Bolonia. }\end{array}$ & $\begin{array}{l}\text { El tutor de carrera: } \\
\text { una figura con "los } \\
\text { pies de barro" }\end{array}$ & $\begin{array}{l}\text { El Plan de Acción } \\
\text { Tutorial: un } \\
\text { complemento del Plan } \\
\text { Bolonia }\end{array}$ & $\begin{array}{c}\text { Alineamiento y } \\
\text { Organización de la } \\
\text { Acción Tutorial en el } \\
\text { contexto de Bolonia }\end{array}$ \\
\hline \multirow{2}{*}{$\begin{array}{l}\text { Los estudiantes } \\
\text { universitarios: } \\
\text { ni tradición ni } \\
\text { información sobre } \\
\text { tutoría. }\end{array}$} & $\begin{array}{l}\text { Los estudiantes, } \\
\text { ajenos a la tutoría }\end{array}$ & $\begin{array}{l}\text { Los estudiantes y su } \\
\text { desinterés hacia la } \\
\text { tutoría }\end{array}$ & $\begin{array}{c}\text { Indiferencia del } \\
\text { estudiante hacia la } \\
\text { tutoría }\end{array}$ \\
\hline & $\begin{array}{l}\text { Los estudiantes dan } \\
\text { una imagen "poco } \\
\text { alentadora". }\end{array}$ & $\begin{array}{l}\text { Actitudes y } \\
\text { Conductas percibidas } \\
\text { en los estudiantes }\end{array}$ & $\begin{array}{c}\text { Los estudiantes } \\
\text { universitarios vistos } \\
\text { por los profesores }\end{array}$ \\
\hline $\begin{array}{l}\text { Significados de } \\
\text { la tutoría para el } \\
\text { profesor }\end{array}$ & $\begin{array}{l}\text { La tutoría académica } \\
\text { centra la atención de } \\
\text { los profesores. }\end{array}$ & $\begin{array}{l}\text { Significados de } \\
\text { la tutoría para el } \\
\text { profesor }\end{array}$ & $\begin{array}{l}\text { La tutoría para el } \\
\text { profesor: entre la } \\
\text { tradición, la creencia } \\
\text { y la obligación }\end{array}$ \\
\hline Ser y Hacer de tutor & & $\begin{array}{l}\text { Significados de Ser y } \\
\text { Hacer de tutor }\end{array}$ & Ser y Ejercer de Tutor \\
\hline $\begin{array}{l}\checkmark \text { El despacho como } \\
\text { lugar de encuentro } \\
\checkmark \text { Distancias y } \\
\text { Cercanías entre } \\
\text { profesores y } \\
\text { estudiantes }\end{array}$ & & & $\begin{array}{l}\text { Distancias entre } \\
\text { profesores y } \\
\text { estudiantes }\end{array}$ \\
\hline
\end{tabular}


Tabla 3

Resultados Caso universidad privada

\begin{tabular}{|c|c|}
\hline CATEGORÍAS & $\begin{array}{l}\text { CASO UNIVERSIDAD } \\
\text { PRIVADA } \\
\text { METACATEGORÍAS }\end{array}$ \\
\hline $\begin{array}{l}\checkmark \text { La Universidad según la mirada de los profesores } \\
\checkmark \text { Discusión sobre el Plan de tutorías de la Universidad }\end{array}$ & $\begin{array}{l}\text { Ser y Ejercer de Profesor } \\
\text { en mi universidad }\end{array}$ \\
\hline $\begin{array}{l}\checkmark \text { Significados de la tutoría para el profesor } \\
\checkmark \text { La tutoría entendida como espacio de comunicación } \\
\quad \text { interpersonal }\end{array}$ & $\begin{array}{c}\text { Los múltiples significados } \\
\text { de la tutoría }\end{array}$ \\
\hline$\checkmark$ Ser y hacer de tutor & Ser y Ejercer de tutor \\
\hline $\begin{array}{l}\checkmark \text { Percepción de los profesores respecto a los estudiantes y la } \\
\text { tutoría } \\
\checkmark \text { La tutoría como espacio de poder del profesor }\end{array}$ & $\begin{array}{c}\text { La tutoría, } \\
\text { un espacio de relación }\end{array}$ \\
\hline
\end{tabular}

El análisis de las diferencias y similitudes entre ambos casos, produjo seis temas transversales:

- Ser y ejercer de profesor en mi universidad.

- Discusión sobre la Reforma en Educación Superior y sus implicaciones en la acción tutorial.

- Los múltiples significados de la tutoría.

- Ser y ejercer de tutor.

- Ser y ejercer de estudiante: la pieza que ignora el sistema.

- La tutoría como espacio docente y de relación.

La heterogeneidad en los resultados obtenidos, responde a una de las características clave de la investigación cualitativa, centrada en indagar sobre las experiencias humanas (De la Cuesta, 2015), además refleja la singularidad de los participantes y la idiosincrasia de cada caso de estudio.

\section{Resultados centrados en la tutoría como espacio docente y de relación}

En este artículo presentamos los resultados relativos a la tutoría como espacio docente y de relación. Se muestran a continuación las relaciones entre categorías y metacategorías, con la frecuencia de verbatim en los códigos que las conforman, acompañados de extractos de las entrevistas con los profesores (Tabla 4). 
Tabla 4

Constructo teórico del tema: la tutoría como espacio docente y de relación

\begin{tabular}{|c|c|c|c|}
\hline CASO & CATEGORÍA & METACATEGORÍA & TEMA \\
\hline \multirow{2}{*}{$\begin{array}{l}\text { Universidad } \\
\text { pública (UPU) }\end{array}$} & $\begin{array}{l}\text { - Distancias y cercanías entre } \\
\text { profesores y estudiantes. } \\
\text { (25 fragmentos, } 3 \text { códigos) }\end{array}$ & \multirow{2}{*}{$\begin{array}{l}\text { Distancias entre pro- } \\
\text { fesores y estudiantes } \\
\text { (32 fragmentos, } 5 \\
\text { códigos) }\end{array}$} & \multirow{5}{*}{$\begin{array}{l}\text { La tutoría como } \\
\text { espacio docente } \\
\text { y de relación } \\
\text { (160 fragmentos, } \\
13 \text { códigos) }\end{array}$} \\
\hline & $\begin{array}{l}\text { - El despacho como lugar de } \\
\text { encuentro } \\
\text { (7 fragmentos, } 2 \text { códigos) }\end{array}$ & & \\
\hline \multirow{3}{*}{$\begin{array}{c}\text { Universidad } \\
\text { privada (UPRI) }\end{array}$} & $\begin{array}{l}\text { - La tutoría entendida como } \\
\text { espacio de comunicación inter- } \\
\text { personal } \\
\text { ( } 57 \text { fragmentos, } 2 \text { códigos) }\end{array}$ & $\begin{array}{l}\text { Los múltiples sig- } \\
\text { nificados de la tutoría } \\
\text { (57 fragmentos, } \\
2 \text { códigos) }\end{array}$ & \\
\hline & $\begin{array}{l}\text { - Percepción de los profesores } \\
\text { respecto a los estudiantes y la } \\
\text { tutoría } \\
\text { (61 fragmentos, } 4 \text { códigos) }\end{array}$ & \multirow{2}{*}{$\begin{array}{l}\text { La tutoría, un espacio } \\
\text { de relación } \\
\text { (71 fragmentos, } \\
6 \text { códigos) }\end{array}$} & \\
\hline & $\begin{array}{l}\text { - La tutoría como espacio de } \\
\text { poder del profesor } \\
\text { (10 fragmentos, } 2 \text { códigos) }\end{array}$ & & \\
\hline
\end{tabular}

\section{Los múltiples significados de la tutoría: un espacio de/para la comunicación}

La tutoría entendida como espacio de comunicación interpersonal, predominó en el caso UPRI. Los profesores se refirieron a la tutoría como un espacio para conversar, de contenido personal y privado, de reflexión y de escucha activa. Un espacio de influencia que va más allá de lo puramente académico:

Olivia: (...) es muy complicado, intentas siempre hablar en un sitio privado, ¿no?, si hay un aula donde te puedes sentar, que hable, que llore, y cuando ya ha hablado y ha llorado intentar siempre desde tu punto de vista, y claro, eso es muy personal, intentar transmitirle sobre todo tranquilidad, y una perspectiva más global (...) (UPRI)

En la UPU, el concepto de tutoría con un sentido académico fue mayoritario, especialmente en el escenario universidad antigua. La categoría "La tutoría académica centra la atención de los profesores" agrupa los códigos referidos a la realización de tutoría académica (Tabla 2).

Los profesores utilizan, en general, facilitadores de la comunicación:

Les recibo en el momento, les escucho (Horacio, UPU antigua), Utilizo la cordialidad, la cercanía, me pongo a su nivel, mostrar tranquilidad, mostrarme un poco paternal, cuidar las palabras que utilizo (Diana, UPU antigua), Trato de empatizar, me gusta conversar (Santiago, UPU nueva), Mostrarse accesible, cercano (Celeste, UPU nueva).

Los participantes del caso UPRI explican sus estrategias comunicativas: establecer relación empática debe ser la principal preocupación, respetar el ritmo del estudiante, no invadir; 
usar lo "no verbal": la mirada y la sonrisa, respetar el espacio y la distancia que desea el otro, "normalizar" situaciones, ayudar a analizar / a ser constructivo, permitir expresión de emociones.

Los profesores centrados en la tutoría estrictamente docente, destacan de forma unánime la importancia del respeto:

Diana:...es importante, que sea desde la cordialidad, y que ellos te respeten porque tú les has demostrado que sabes las cosas, que tienes seguridad, pero no porque tengas más poder porque seas más fuerte, y entonces te vendrán más (UPU antigua)

El profesor busca la privacidad en la tutoría, relacionándolo con necesidades de los estudiantes:

Olivia: porque son cosas personales lo que te quieren decir, entonces al final de una clase, en un patio, un pasillo, en la policlínica... ¿cuándo puedo hablar con usted?, (...) es muy complicado, intentas siempre llevarlos a un sitio privado, ¿no? (UPRI)

\section{Percepción de los profesores respecto a los estudiantes y la tutoría}

En el caso UPRI hablaban de conductas, emociones y actitudes de los estudiantes, reconocidas durante las tutorías, y que reflejan contenidos conversacionales:

Olivia: ... y veo mucha diferencia de madurez, como afrontan o no los problemas, y como te los trasladan, y el tipo de problema que tienen no tiene nada que ver. Los estudiantes de cursos superiores cuentan cosas más concretas, más reales, menos de emoción personal ¿no? (UPRI)

\section{Distancias entre profesores y estudiantes}

Esta metacategoría reunió significados sobre cómo son las relaciones establecidas entre profesores y estudiantes, en ambos casos de estudio.

Horacio: A lo mejor hay profesores que tengan un aspecto más autoritario, que guarden más las distancias con los alumnos, a lo mejor estos se retraen a la hora de preguntarle, (...) además, a veces notan muy poca respuesta por parte de algunos profesores en esas tutorías... (UPU antigua)

Santiago: (...) Y yo creo que lo fundamental para mí en la tutoría es la cercanía que tenga el profesor a la hora de poder resolver esa tutoría, lo accesible que sea... que el alumno pocas veces acude si no ve esa cercanía, (UPU nueva)

Durante la tutoría, se normaliza la existencia de límites por parte del profesor, y el establecimiento de distancias que se consideran necesarias entre tutor y estudiante:

Tirso: parece que en mis tutorías hay un cierto límite, ¿no?,(...) quizá no es propicio que alguien traiga un café o que me llame colega o, hay una relación de respeto muy cordial, y casi informal, pero se habla de un tema y hay alguien que pregunta y alguien que responde (UPRI)

Olivia: (...) no me veían como a un profesor, entonces creo que hay que marcar esa distancia, pero siempre dando, dejando la puerta abierta de la cercanía (UPRI)

Los participantes identifican elementos en el profesorado, que no favorecen el acercamiento de los estudiantes, o les hacen sentir incómodos en distancias cortas. Algunos rasgos genéricos distintivos en los profesores distantes son:

- Utilizar el "usted" para hablar con los estudiantes.

- Trato brusco o cortante. 
- Al relacionarse parece que estuvieran "en un escalón más alto".

- Se sienten molestos ante solicitud de consulta de dudas o problemas.

- Producen sensación de que se les está "robando el tiempo".

\section{La tutoría como espacio de poder del profesor}

En el espacio de la tutoría, se identificaron símbolos del saber que representan el poder del profesor (Tabla 5):

Úrsula: Depende de la formación y forma de ser del profesor, ya que hay mucha resistencia en ocasiones a dejar el protagonismo al alumno y "perderlo" nosotros. (UPRI)

Celeste: Yo creo que el alumno ve al profesor, como en un escalón más arriba, aunque quizás va cambiando poco a poco, (UPU nueva)

Tabla 5

Símbolos del Saber cómo Poder, identificados en el discurso de los profesores

SÍMBOLOS DEL SABER

LA TARIMA

LA CLASE MAGISTRAL

LA ACTIVIDAD INVESTIGADORA

TITULACIÓN DE PRESTIGIO
Virginia: ¡Respétenme a mí!, que estoy aquí en la tarima (UPU)

Úrsula: No todos los profesores pueden evitar no dar de nuevo una clase magistral en una tutoría académica (UPRI)

Diana: El prepotente profesor de la universidad pública, da su clase y luego es un investigador (UPU)

Tirso: En tutoría tienen un médico, un ingeniero (...) eso es un lujo (UPRI)

\section{El despacho como lugar de encuentro}

El despacho, o el uso de espacios compartidos por varios profesores, no facilitan la relación cercana y genera incomodidad en los estudiantes.

Celeste: no hay sala habilitada como tal para tutoría, ni creo que se piense, (...) A lo mejor meterte en el despacho del profesor es algo como que te da respeto (UPU nueva)

\section{Discusión y conclusiones}

La literatura en el contexto español, es abundante y diversa en las dos últimas décadas; sin embargo, los estudios sobre lo que sucede en la tutoría, cómo se produce la relación entre tutor y estudiante, las estrategias comunicativas que utilizan los tutores, y los factores que modulan esta relación, son aspectos menos abordados.

Respecto al primer objetivo, la tutoría debe fundamentarse en la comunicación y en la relación personal cercana; ambas cuestiones son el instrumento primordial para 
desempeñar la función de tutelaje. Mientras que los profesores del caso UPU utilizan facilitadores de la comunicación de forma más intuitiva, en el caso UPRI utilizan estrategias de comunicación de forma intencional, aportando argumentos a favor del valor de la escucha, los elementos no verbales y la confidencialidad. Este enfoque coincide con Haya, et al. (2013), López-Gómez (2016) y Martínez, Pérez, et al. (2014), al expresar que la tutoría debe constituir una interacción bidireccional, una relación de ayuda, donde existe un compromiso que asumen profesor y estudiante desde la confianza, construyendo un clima relacional propicio para la comunicación. Supone el establecimiento de una "corriente de empatía", necesaria sea cual sea el objetivo y contenidos del encuentro (Fernández-Salinero, 2014). Investigaciones sobre la satisfacción de los estudiantes con el plan tutorial de su titulación, muestran la importancia de la dimensión personal, siendo este el aspecto más valorado por los estudiantes (Torrecilla et al., 2013), y también reconocido por los profesores (Haya et al., 2013; Prieto, 2015).

¿Cuáles son las barreras u obstáculos que están impidiendo que los espacios de tutoría ocupen un lugar central en el modelo docente de las universidades, y por tanto en la formación y desarrollo de los estudiantes?

Respecto al segundo objetivo, se observa que la distancia establecida por el tutor puede ser un obstáculo en la comunicación y condiciona que los estudiantes acudan a tutoría. Esto coincide con Pantoja y Campoy (2009), que aluden a la importancia del estilo del tutor, si es dialogante e integrador; o por el contrario, es directivo y distante, lo cual promueve o dificulta las relaciones con sus estudiantes.

Según los participantes, los profesores distantes muestran su rechazo o indiferencia hacia la tutoría en diferentes formas, que son evidentes para otros profesores y para los estudiantes. La distancia establecida por el tutor, la actitud autoritaria, puede ser un obstáculo en la comunicación, y en definitiva, condiciona la decisión de estos, a la hora de solicitar tutoría.

Otras barreras según Martínez, Martínez, et al. (2014), son la falta de información en los estudiantes, que además desconocen los beneficios que se pueden obtener al utilizar la tutoría universitaria, la falta de tradición y formación de los docentes en la función tutorial, el escaso reconocimiento institucional frente a otras funciones como la investigadora, y la consideración de los tiempos de tutoría como tareas secundarias dentro de la actividad docente (Prieto, 2015). Todos ellos, son elementos desmotivadores, que obstaculizan la labor tutorial. Sin embargo, en los casos de estudio se identifica que lo esencial en los tutores es una actitud de mayor compromiso y relación de confianza, coincidiendo con lo que demandan los estudiantes (Martínez, Martínez, et al., 2014). Los profesores destacan que deben estar presentes en la tutoría, el respeto mutuo y la privacidad para tratar temas personales. Monroy (2013), explica que la estima, el respeto, la confianza recíproca, preserva la relación simétrica, aun en relaciones asimétricas, como profesor-estudiante o padres-hijos.

Los participantes coinciden en que es necesario establecer ciertos límites para no perder el status de profesor, aunque expresan no saber cuáles o cómo establecerlos. Algunos prefieren la tarima, utilizan un trato distante, priorizan la función investigadora y mantienen una actitud más jerárquica. Son elementos que caracterizan una concepción clásica del profesor. Los roles de transmisor de conocimientos y de alumnos pasivos están muy arraigados en nuestra cultura universitaria (Pantoja \& Campoy, 
2009; Prieto, 2015); y ello se traduce en relaciones distantes. La relación entre profesor y alumno está llena de poder y estatus (Taibi, 2006). Según Prieto (2015), los profesores les concedían bajas puntuaciones a las tutorías personales, frente a las académicas, porque les generaba miedo traspasar los límites con el estudiante, convirtiéndose en un colega, perdiendo status de docente.

La resistencia al cambio de modelo docente, y a métodos en los cuales el profesor debe ceder el protagonismo, sitúa a algunos profesores en una posición incómoda y resistente respecto a la tutoría integral. Esto explicaría que siga enfocándose hacia la tutoría de tipo académico, un espacio de confort y control mediante el conocimiento experto. La tutoría es un espacio privado, sin espectadores, donde la autoridad del profesor, investida por los "símbolos del saber", queda difuminada.

Sobre la identificación de elementos para el desarrollo y mejora de la acción tutorial universitaria, los resultados apuntan:

- La necesidad de que las universidades articulen formas de promover cultura tutorial en todos los actores, de forma que ésta se incorpore como pieza crucial al engranaje del EEES.

- La universidad privada, más flexible en sus funcionamientos, parece estar desarrollando con menos trabas una acción tutorial más personalizada, donde lo académico y lo personal se integran. Las universidades públicas deberían asumir estructuras y funcionamientos más flexibles, acordes con la filosofía del EEES (Lázaro, 2002).

- Los perfiles de los profesores-tutores deben adecuarse a las necesidades y tipo de demandas de los estudiantes. La motivación y formación del profesorado es fundamental y la implicación de las instituciones, indispensable.

- La falta de reconocimiento de la función tutorial va en claro detrimento de la materialización de un modelo de tutoría sólido e integrado en la función docente del profesorado.

- La tutoría entre iguales facilita una relación más simétrica y horizontal (Flores, Gil \& Caballer, 2012), pero todavía está poco desarrollada en España (Álvarez González \& Álvarez Justel, 2015; Prieto, 2015). Universidades de otros países como Francia, Reino Unido, Canadá y Estados Unidos, optan por un modelo de tutoría entre iguales o peermentoring para los alumnos de primeros años, además de la figura del profesor-tutor.

En conclusión, si bien profesores y estudiantes están de acuerdo en el valor de la tutoría en general, y en la necesidad de reorientarla para situar al estudiante en el centro, se identifican obstáculos para que este cambio se haga realidad. El acercamiento del alumnado a la tutoría, dependerá en gran medida del estilo de comunicación y la forma de relacionarse del tutor, es decir, de su capacidad para establecer una relación cercana y más horizontal con el estudiante. Cuanto menos asimétrica y jerárquica es la relación tutor-estudiante, más efectivo puede ser el aprendizaje y más impregnará la tutoría en el alumnado. Las relaciones basadas en la confianza, el respeto mutuo, y una formación y motivación sólida del profesor respecto a la función tutorial, pueden favorecer la tutoría de carácter personal frente a la estrictamente académica. 


\section{Limitaciones y algunas líneas de investigación}

Aunque se han llevado a cabo métodos de triangulación, en investigación cualitativa el muestreo es no aleatorio, con lo cual, la motivación en los profesores, el área de conocimiento, etc., podrían constituir un tipo de sesgo de selección de muestra. Es necesario interpretar los resultados en cada caso dentro del contexto histórico y particular que caracteriza a ambos tipos de universidad, considerando la validez interna propia de los estudios cualitativos. Es conveniente seguir investigando para conseguir elementos de mejora que promuevan un cambio real en el desarrollo de la tutoría, incorporar estudiantes y gestores académicos como muestras de estudio y contrastar con investigaciones procedentes de otras universidades extranjeras con mayor tradición tutorial.

\section{Referencias}

Albanaes, P., Marques de Sousa, F., \& Patta M. (2015). Programas de tutoría y mentoría en universidades brasileñas: un estudio bibliométrico. Revista de Psicología, 33(1), 21-56. Recuperado de http://revistas.pucp.edu.pe/index.php/psicologia/article/wiew/11170

Álvarez González, M., \& Álvarez Justel, J. (2015). La tutoría universitaria: Del modelo actual a un modelo integral. Revista Electrónica Interuniversitaria de Formación del Profesorado, 18(2), 125-142. doi: 10.6018/reifop.18.2.219671

Atkinson, P., \& Hammersley, M. (2008). Etnografía. Métodos de investigación. Barcelona: Paidós Ibérica.

Coller, X. (2000). Estudio de casos: Cuadernos metodológicos. Madrid: Centro de Investigaciones Sociológicas.

De Jorge, J., Gil, L., Merino, F., \& Sanz, M. (2011). Evidencia empírica de los motivos del absentismo en los estudiantes universitarios. Revista de Investigación en Educación, 2(9), 76-90. Recuperado de https://dialnet.unirioja.es/servlet/articulo?codigo $=4731292$

De la Cuesta, C. (2015). La calidad de la investigación cualitativa: de evaluarla a lograrla. Texto \& Contexto Enfermagem, 24(3), 883-890. doi: 10.1590/0104-070720150001150015

Fernández, G. M., \& Escribano, M. C. (2009). Las tutorías en la formación académica y humana de los alumnos en la Universidad San Pablo CEU. Actas de las XVI Jornadas ASEPUMA - IV Encuentro Internacional 2008, 16. Recuperado de http://metodos. upct.es/asepuma/comunicaciones/completas /605.pdf

Fernández-Salinero, C. (2014). La tutoría universitaria en el escenario del Espacio Europeo de Educación Superior: Perfiles actuales. Teoría de la Educación. Revista Interuniversitaria, 26(1), 161-186. doi: 10.14201/teoredu2014261161186

Figuera, P., \& Álvarez, M. (2014). La intervención orientadora y tutorial en la adaptación y persistencia del alumnado en la universidad. Revista de Orientación Educacional, 28(54), 31-49. Recuperado de http://www.roe.cl/index.php/roe/article/view/83

Flores, R., Gil, J. M., \& Caballer, A. (2012). Rol del Servicio de Orientación en universidades de Francia, EE. UU., Canadá, Reino Unido y España. Papeles del Psicólogo, 33(2), 138-147. Recuperado de http://hdl.handle.net/10234/62932

García Fernández, A. (2006). La tutoría y la relación profesor-alumno en la formación para la inserción laboral. Revista de Educación, 341, 197-211. Recuperado de http:// www.revistaeducacion.mec.es/re341/re341_09.pdf 
García González, A. J., \& Troyano, Y. (2009). El Espacio Europeo de Educación Superior y la figura del profesor tutor en la universidad. Revista de Docencia Universitaria, 3, Recuperado de http://revistas.um.es/redu/article/view/69991

García Nieto, N. (2008). La función tutorial de la universidad en el actual contexto de la Educación Superior. Revista Interuniversitaria de Formación del Profesorado, 22(1), 21-48. Recuperado de: http://www.redalyc.org/articulo.oa?id=27413170002

García-Nieto, Asensio, I., Carballo, R., García-García, M., \& Guardia, S. (2005). La tutoría universitaria ante el proceso de armonización europea. Revista de Educación, 337, 189-210. Recuperado de http://www.revistaeducacion.mec.es/re337/re337_10.pdf

García-Cabrero, B., Ponce, S., García, M.H., Caso, J., Morales, C., Martínez Y., ... Aceves, Y. (2016). Las competencias del tutor universitario: una aproximación a su definición desde la perspectiva teórica y de la experiencia de sus actores. Perfiles Educativos, 38(151), 104-122. Recuperado de http://www.scielo.org.mx /scielo.php?script=sci_art text\&pid=S0185-26982016000100104

Giménez, S. (2007). Construyendo el espacio europeo de educación superior: La tutoría personalizada como acompañamiento formativo. Miscelánea Comillas, 65(127), 689705. Recuperado de http://revistas.upcomillas.es/index.php/miscelaneacomillas/ article/view/7330

Glaser, B. G., \& Strauss, A. L. (2009). The discovery of grounded theory: Strategies for qualitative research. Transaction publishers.

Goodman, L. A. (2011). Comment: On respondent-driven sampling and snowball sampling in hard-to-reach populations and snowball sampling not in hard-to-reach populations. Sociological Methodology, 41(1), 347-353. doi: 10.1111/j.1467-9531.2011.01242.x

Haya, I., Calvo, A., \& Rodríguez, C. (2013). La dimensión personal de la tutoría universitaria: Una investigación cualitativa en la Universidad de Cantabria (España). Revista Española De Orientación y Psicopedagogía, 24(3), 98-113. Recuperado de http://e-spacio. uned.es/fez/eserv/bibliuned:revistaREOP-2013-24-3-7060/Documento.pdf

Lázaro, A.J. (2002). La acción tutorial de la función docente universitaria. Calidad de las universidades y orientación universitaria. En A. J. Lázaro \& V. Álvarez Rojo (Coords.), Calidad de las universidades y orientación universitaria (pp. 249-282) Ediciones Aljibe.

Lázaro, A. J. (2008). Diferencias cualitativas entre experiencias tutoriales para opciones de aprendizaje universitario. Revista Interuniversitaria de Formación del Profesorado, 22(1), 109-137. Recuperado de: http://www.redalyc.org/articulo.oa?id=27413170006

López-Gómez, E. (2015). La tutoría en el EEES: Propuesta, validación y valoración de un modelo integral. (Tesis Doctoral, UNED). Recuperado de http://e-spacio.uned.es/fez/ view/tesisuned:Educacion-Elopez

López-Gómez, E. (2016). La tutoría universitaria como relación de ayuda. Opción, 32(9), 1007-1024. Recuperado de http://www.produccioncientifica.luz.edu.ve/index.php/ opcion/article/view/21789

López-Martín, I. (2012). Significados de la tutoría universitaria desde la percepción de los profesores. (Tesis Doctoral, Universidad Europea de Madrid). Recuperado de http:// hdl.handle.net/11268/1341

López-Martín, I., González, P., \& Velasco P.J. (2013). Ser y Ejercer de tutor en la universidad. Revista de Docencia Universitaria, 11(2), 107-134. Recuperado de http://red-u. net/redu/index.php/REDU/article/download/581/pdf? 
Martínez, P.C. (2006). El método de estudio de caso estrategia metodológica de la investigación científica. Revista Científica Pensamiento y Gestión, 20, 165-193. Recuperado de http://www.redalyc.org/articulo.oa?id=64602005

Martínez Clares, P., Martínez Juárez, M., \& Pérez Cusó, J. (2014). Tutoría Universitaria: entorno emergente en la Universidad Europea. Un estudio en la Facultad de Educación de la Universidad de Murcia. Revista de Investigación Educativa, 32(1),111-138. doi: http://dx.doi.org/10.6018/rie.32.1.148411

Martínez Clares, P., Pérez Cusó, J., \& Martínez Juárez, M. (2014) Una (re)visión de la tutoría universitaria: la percepción de estudiantes y tutores de estudios de Grado. REDU. Revista de Docencia Universitaria, 12(1), 269-305. doi:https://doi. org/10.4995/redu.2014.6425.

Mena-Rodríguez, E. (2015). El comportamiento interpersonal de los mejores docentes en las aulas de la universidad. Estudio cuantitativo por ramas de conocimiento. En Asociación Interuniversitaria de Investigación Pedagógica -AIDIPE (Eds.), Investigar con y para la sociedad (Vol. 1, pp. 359-368). Cádiz, España: Bubok. Recuperado de http://aidipe2015.aidipe.org

Monroy, V. (2013). Las identidades profesionales de los docentes principiantes desde la intencionalidad ética. Revista Mexicana De Investigación Educativa, 18(58), 789-810. Recuperado de http://www.scielo. org.mx/scielo.php?script=sci_arttext\&pid=S1405 66662013000300006\&lng=es\&tlng=es.

Pantoja, A., \& Campoy, T. J. (2009). Planes de acción tutorial en la universidad. Jaén: Universidad de Jaén.

Paricio, J. (2005). Objetivos y contenidos de la acción tutorial en el ámbito de las titulaciones universitarias. Zaragoza, España: Instituto de Ciencias de la Educación, Universidad de Zaragoza.

Prieto, N. (2015). La tutoría en el Espacio Europeo de Educación Superior. Visión del profesorado. (Tesis Doctoral, Universidad de La Coruña). Recuperado de https://dialnet. unirioja.es/servlet/tesis?codigo $=46540$

Rodríguez Espinar, S. (Coord.). (2004) Manual de tutoría universitaria. Recursos para la acción. Barcelona: Octaedro.

Rodríguez-Gómez, G., Gil-Flores, J., \& García-Jiménez, E. (1999). Metodología de la investigación cualitativa. Málaga: Ediciones Aljibe.

Rodríguez-Hoyos, C., Calvo A., \& Haya, I. (2015). La tutoría académica en la Educación Superior. Una investigación a partir de entrevistas y grupos de discusión en la Universidad de Cantabria (España). Revista Complutense de Educación, 26(2), 467-481. doi: 10.5209/rev_RCED.2015.v26.n2.43745

Romero, C., Ortega, F., \& Molina, F. (2016). La autonomía y orientación en el Espacio Europeo de Educación Superior mediante el portafolio y la tutoría. Estudios sobre Educación, 19, 261-282. Recuperado de http://www.unav.edu/publicaciones/revistas/ index.php/estudios-sobre-educacion/ article/view/4622

Ruiz Olabuénaga, J. I. (2012). Metodología de la investigación cualitativa. Bilbao: Universidad de Deusto.

Sandín, M. P. (2010). Investigación cualitativa en educación: Fundamentos y tradiciones. Madrid: McGraw-Hill.

Stake, R. E. (1999). Investigación con Estudio de Casos. Madrid: Morata. 
Strauss, A. L., \& Corbin, J. (2002). Bases de la investigación cualitativa: Técnicas y procedimientos para desarrollar la teoría fundamentada. Medellín: Universidad de Antioquia.

Taibi, M. (2006). Reconsidering tutorials and student-lecturer power relationships in language subjects. Porta Linguarum: Revista Internacional De Didáctica De Las Lenguas Extranjeras, 6, 23-32. Recuperado de https://dialnet.unirioja.es/servlet/ articulo? codigo $=2371484$

Taylor, S., \& Bogdan, R. (2009). Introducción a los métodos cualitativos de investigación. Barcelona: Paidós.

Torrecilla, E. M., Rodríguez, M. J., Herrera, M. E., \& Martín, J. F. (2013). Evaluación de calidad de un proceso de tutoría de titulación universitaria: La perspectiva del estudiante de nuevo ingreso en educación. REOP, 24(2), 79-99. doi: 10.5944/reop. vol.24.num.2.2013.11260

Vallés, M.S. (2009). Entrevistas Cualitativas. Cuadernos Metodológicos nº 32. Madrid: CIS

Velasco, H. M., \& Díaz de Rada, A. (2003). La lógica de la investigación etnográfica: Un modelo de trabajo para etnógrafos de la escuela. Madrid: Trotta.

Zabalza, M. A. (2003). Competencias docentes del profesorado universitario: Calidad y desarrollo profesional. Madrid: Narcea Ediciones.

Fecha de recepción: 8 de abril de 2017

Fecha de revisión: 15 de abril de 2017

Fecha de aceptación: 4 de abril de 2018 
